\title{
UV illumination for electron and ion beam microscopy and nanofabrication
}

\author{
Soon Hock Nga,b ${ }^{\mathrm{a}}$ Gediminas Seniutinas ${ }^{\mathrm{c}, \mathrm{a}}$, Meguya Ryu ${ }^{\mathrm{d}}$, Tomas Katkus ${ }^{\mathrm{a}}$, \\ Nguyen Hoai An Le ${ }^{a}$, Paul Stoddart ${ }^{\mathrm{e}}$, Junko Morikawa ${ }^{\mathrm{d}}$, and Saulius Juodkazis ${ }^{\mathrm{a}, \mathrm{b}, \mathrm{f}}$ \\ ${ }^{a}$ Centre for Micro-Photonics, Faculty of Science, Engineering and Technology, Swinburne \\ University of Technology, Hawthorn, Victoria 3122, Australia \\ b Melbourne Centre for Nanofabrication, 151 Wellington Rd., Clayton, Victoria 3168, Australia. \\ ${ }^{\mathrm{c}}$ Laboratory for Micro and Nanotechnology, Photon Science Division, Paul Scherrer Institut, \\ Forschungsstrasse 111, 5232 Villigen PSI, Switzerland \\ dSchool of Materials and Chemical Technology, Tokyo Institute of Technology, 2-12-1 \\ Ookayama, Meguro-ku, Tokyo 152-8550, Japan \\ ${ }^{\mathrm{e} C e n t r e ~ f o r ~ Q u a n t u m ~ a n d ~ O p t i c a l ~ S c i e n c e, ~ F a c u l t y ~ o f ~ S c i e n c e, ~ E n g i n e e r i n g ~ a n d ~ T e c h n o l o g y, ~}$ \\ Swinburne University of Technology, Hawthorn, Victoria 3122, Australia \\ ${ }^{\mathrm{f}}$ Tokyo Tech World Research Hub Initiative (WRHI), School of Materials and Chemical \\ Technology, Tokyo Institute of Technology, 2-12-1, Ookayama, Meguro-ku, Tokyo 152-8550,
} Japan

\begin{abstract}
The scanning electron microscope (SEM) has become the major imaging technique of choice over many fields of science, providing resolution down to a few nanometers. To reduce surface charging, low beam currents and a few-nm-thick highly conductive metal (Au, Pt) coatings are used. The conceptual problem with the metal coating is that it conceals the sample features which it aims to reveal and the characterised samples are altered. A novel approach to control surface charging in SEM via the photoelectric effect is shown. The technique uses deep-UV co-illumination during SEM imaging. Photons of the deep-UV light have sufficient energy to liberate electrons from the sample surface reducing strong charge gradients. In addition, the method provides a new material dependent contrast modality in SEM. Deep-UV illumination also improves nanoscale 3D structuring using focused ion beam milling. Instead of the metal coating for electron/ion imaging, the newly introduced "coating by light" is introduced. Load-lock mountable sample holder with a deep-UV multiwavelength illumination is conceptualised.
\end{abstract}

Keywords: charge removal, electron work function, UV light emitting diode, SEM Stage

\section{INTRODUCTION}

Electron and ion particle beams are ubiquitous in both high-resolution imaging (e.g. scanning electron microscope (SEM) or transmission electron microscope (TEM)) and lithography (e.g. electron beam lithography (EBL) or focused ion beam (FIB)) applications. In both cases, surface charging of the substrate during irradiation can cause distortion of the beam path, manifesting as distorted images or patterns. ${ }^{1}$ This is particularly problematic with insulating materials as the charge is not able to dissipate through the substrate.

The most common method to mitigate charge is the addition of a conductive layer. For imaging, this usually involves sputtering a thin layer of gold or platinum on the samples surface. The conceptual problem with the metal coating is that it conceals the sample features which it aims to reveal and can destroy the functionality of a device, such as with micro lenses or ring resonators. For EBL, a thin Cr layer is deposited over (or under) the resist and has to be removed before development or downstream processing. In this case, it adds an extra

Further author information: (Send correspondence to S.-H. Ng and S. Juodkazis)

E-mails: soonhockng@swin.edu.au (S.-H. N.); sjuodkazis@swin.edu.au (S. J.)

UV and Higher Energy Photonics: From Materials to Applications 2019, edited by Gilles Lérondel,

Yong-Hoon Cho, Atsushi Taguchi, Satoshi Kawata, Proc. of SPIE Vol. 11086, 110860U

(c) 2019 SPIE · CCC code: 0277-786X/19/\$21 - doi: 10.1117/12.2528681

Proc. of SPIE Vol. $11086110860 \mathrm{U}-1$ 

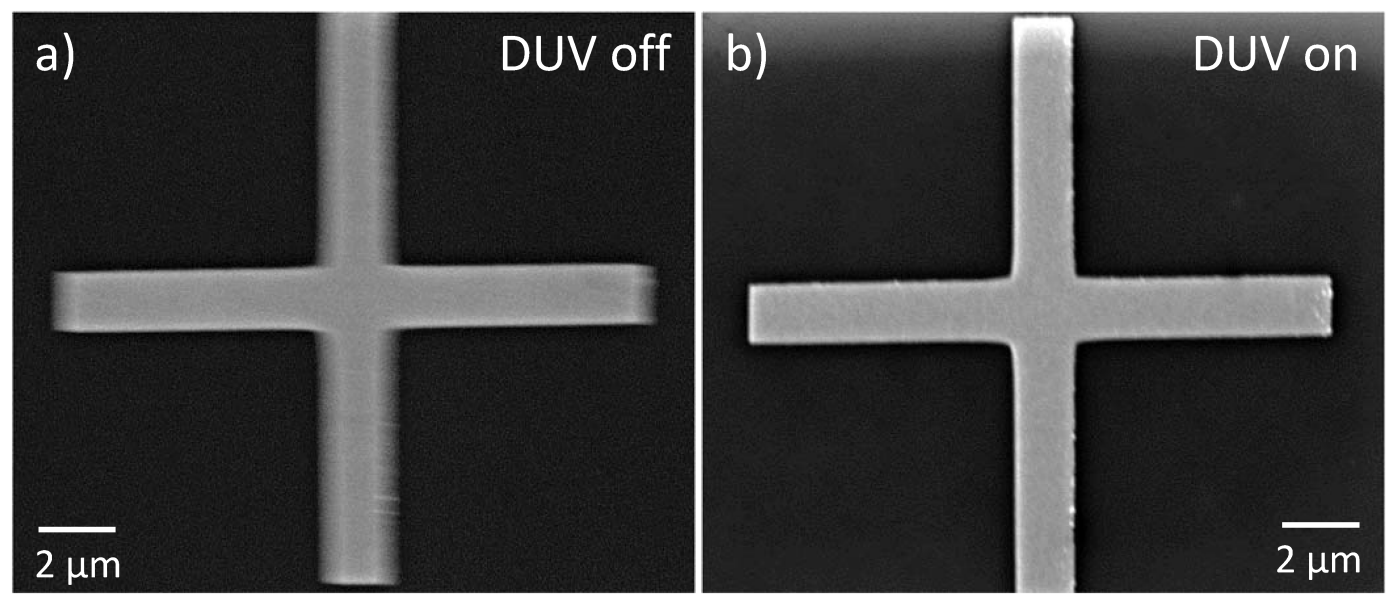

Figure 1. Surface charging reduction via DUV illumination. SEM images of gold cross-like structure on silicon substrate taken without (a) and with (b) DUV illumination. Both images taken by integrating 16 frames to emphasize the blurring caused by surface charging. The imaging electron beam voltage and current were set at $10 \mathrm{keV}$ and $60 \mathrm{nA}$, respectively.

processing step as well as possible beam scattering at lower accelerating voltages. Beam current in SEM can be reduced to slow the rate of surface charging, which has the benefit of increasing resolution due to the smaller beam spot size. Conversely, image quality is reduced due lower signal to noise or a longer image acquisition time, where the sample could move and distort the image.

A novel approach to control surface charging via the photoelectric effect has previously demonstrated for both SEM and FIB lithography by our group. ${ }^{2,3}$ It utilises UV LED to irradiate the substrate alongside the charged particle beam (co-illumination) in the vacuum chamber. Sub-surface defects are formed in dielectrics under electron or ion-beam irradiation, which can have trapping energies up to $0.5 \mathrm{eV}$ for electrons and holes. ${ }^{2,4,5}$ Absorption of UV light also creates defects caused by exciton self-trapping. ${ }^{6}$ For semiconductors and dielectrics, all these defects can be represented as range of surface states in the band diagram for the material. ${ }^{4}$ Hence, the energy required to free the electrons to the vacuum level is slightly reduced in these defects when compared to the material work function $(\phi)$. Deep-UV (DUV) photons have sufficient energy to liberate electrons from the sample, reducing surface charging and hence distortions resulting from it.

Here, we further developed characterisation by the proposed method using slow scanning vs frame averaging in SEM imaging. A concept for load-lock mountable UV-lamp on a sample holder is presented, considering the typical geometry of SEM microscope and electron beam lithography (EBL) chamber of a Raith $150^{\text {TWO }}$ tool. The proposed stand alone sample holder with an UV lamp illumination during SEM/TEM/FIB imaging or nanofabrication can be make compatible on tools from different vendors.

\section{EXPERIMENTAL}

A simple homemade sub-stage was fabricated from aluminium moulding. ${ }^{2,3}$ A UV LED (SETi Ltd.) with peak wavelength of $260 \mathrm{~nm}(4.77 \mathrm{eV})$ was mounted at $60^{\circ}$. The sample was attached to the sub-stage using doublesided Kapton tape to enhance the charging effect by isolating it from grounding. This sub-stage was mounted onto 07-09v4 USH stage for a Raith $150^{\text {TWO }}$ electron beam lithography tool. In our first experiments, ${ }^{2,3}$ the stage assembly was too tall to be loaded through the load-lock. Therefore the vacuum chamber had to be opened. Since electrical connections need to be connected to feed-through contacts in order to control the LED from outside, we used service connectors and wiring under advice from the Raith engineering team. The chamber was left to pump down overnight. A film of $50 \mathrm{~nm}$ gold was deposited on both $\mathrm{TiO}_{2}$ and silicon and samples were imaged with an accelerating voltage of $10 \mathrm{kV}$. Using this procedure, the SEM imaging mode was tested with different image acquisition methods: long exposure vs averaging of large number of frames acquired fast. Finally, a new design of sample stage which can be loaded through the load-lock is presented in this work. 

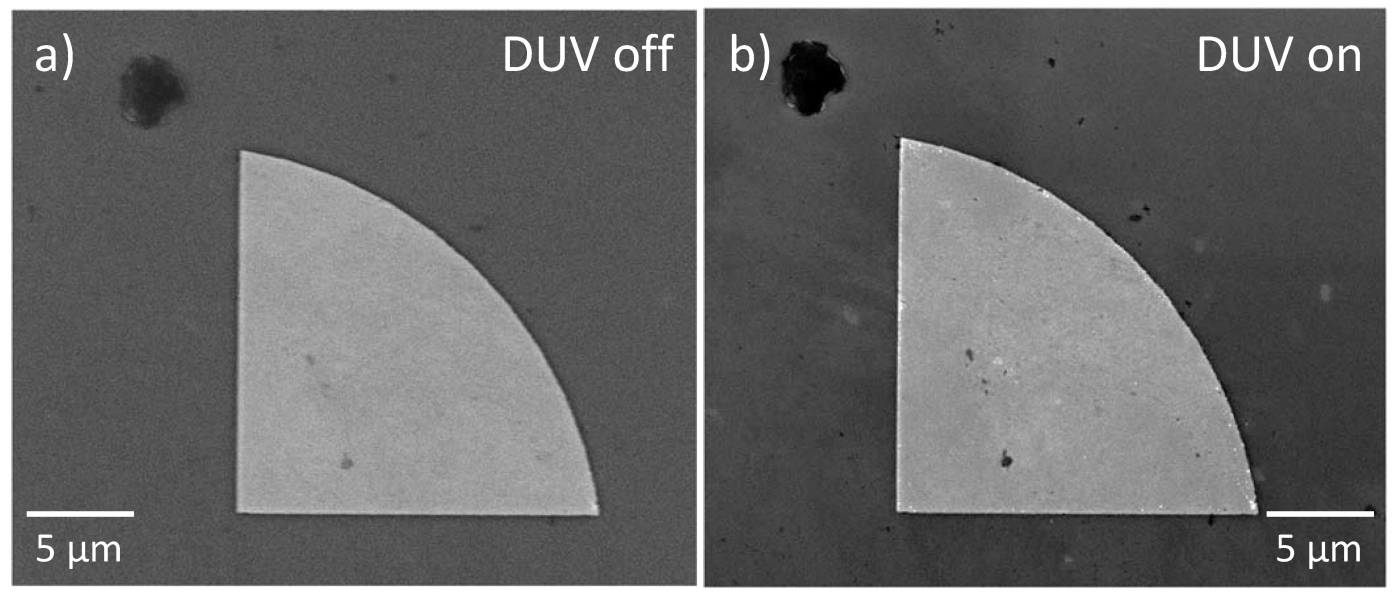

Figure 2. SEM images of a gold pad on $\mathrm{TiO}_{2}$ substrate taken without (a) and with (b) DUV illumination. In both cases, brightness and contrast values were adjusted during the imaging to better reveal the surface details.

\section{RESULTS AND DISCUSSION}

Figure 1 shows a gold $(\phi=5.1-5.47 \mathrm{eV})$ cross on a silicon $(\phi=4.60-4.85 \mathrm{eV})$ substrate with the LED off (a) and on (b). The quality of the DUV illuminated image is significantly better than the electron only image, with sharper edges and better contrast. Both images were taken with an accelerating voltage of $10 \mathrm{kV}$, beam current of $60 \mathrm{nA}$ and is the result of integrating 16 frames to exaggerate the drift caused by sample charging. With DUV on, the accumulated electrons are able to escape the surface, eliminating the drift caused by surface charging. Fast scans under UV illumination does not deteriorate the quality of image, which is a common issue in standard SEM imaging, where slow acquisition has to be used for better quality.

Figure 2 shows a gold pad on a $\mathrm{TiO}_{2}\left(\phi=4.9-5.5 \mathrm{eV}^{7}\right)$ substrate with the LED off (a) and on (b). In each case, brightness and contrast was adjusted to maximise surface detail. It is evident that greater surface detail and contrast is achieved with DUV on. Even though both gold and $\mathrm{TiO}_{2}$ have higher work functions than the incident photons, defect levels and electron repulsion (due to charge build-up) mean that some electrons require lower energy to be excited to vacuum and hence the DUV light still has a significant effect on the image quality. Particles of residual of PMMA resist used in EBL exposure to define the pattern are recognisable as smal dark features. They are less charged so therefore appear darker in SEM image with electrons excited from surface traps.

Not shown here, but the proposed UV co-illumination during SEM imaging should help to reach high resolution $\sim 1 \mathrm{~nm}$ imaging with material selectivity (by electron work function). For wider applications of this method, it is necessary to develop versatile sample stage with capability to wavelength tailor UV illumination during SEM, TEM, or FIB. Figure 3 shows concept of such UV lamp on sample stage which can be used through standard load lock gate. Since DC power is used in LEDs it is a low likelihood that UV illumination will intervene with SEM imaging. We have tested this method on the field-emission Raith EBL tool as well as FIB and have not encountered any cross talk with electron imaging.

\section{CONCLUSIONS AND OUTLOOK}

A simple DUV LED based charge mitigation method was reported which eliminates the need for conductive coatings on electrically isolated samples while also providing enhanced surface detail during SEM imaging. When used in combination with high-resolution SEM, it has the potential to facilitate imaging of nanostructures that are on the same length-scale as the aforementioned conductive coating thickness. Its non-destructive nature means that sensitive devices can be inspected and still be used in downstream processes.

UV co-illumination can help to directly measure the surface charging influence on a direct measurement of surface temperature by a microthermocouple under electron beam excitation. We recently demonstrated a fast 

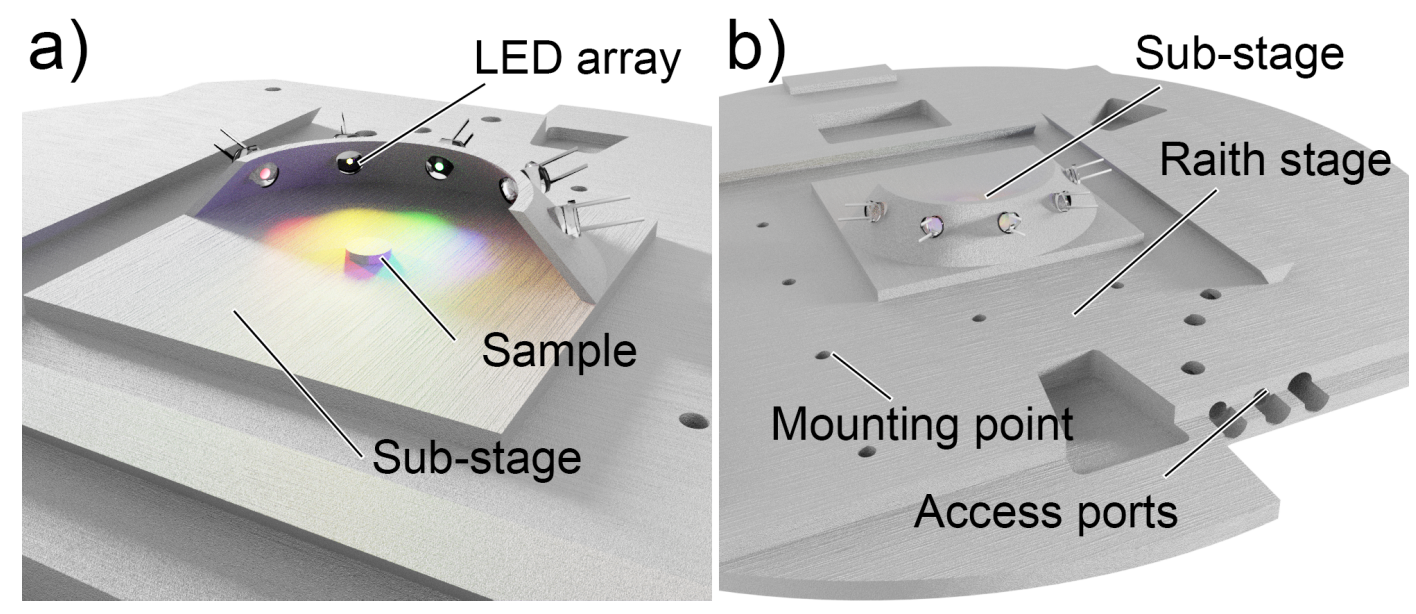

Figure 3. Schematic render of (a) the sub-stage with LED array concept showing LEDs with different wavelengths (colours exaggerated to show this) and (b) the mounting points on the Raith stage as well as access ports for power feed through.

detection of temperature changes induced by electron beam on a nanomembrane. ${ }^{8}$ For direct measurement of thermal diffusion and localisation by electron beam, it is necessary to reduce surface charging which effectively changes the thermopower of materials used for the thermocouple.

Before any of this becomes reality, the major hurdle that needs to be overcome is installation. As described in Section 2, the entire vacuum chamber had to be opened for installation. To maintain high vacuum, any components mounted on the stage must be transferred through the Raith load-lock. A conceptual rendering of an improved sub-stage is shown in Fig.3a. The stage consists of an LED array with a mounting area for the sample. The array as shown here has LEDs of different wavelength in each location. Such a configuration can be used to explore the effects of photon energy on the image characteristics.

Figure $3 \mathrm{~b}$ shows the location of mounting points and access ports which will be used to attach the sub-stage onto the Raith stage and connect it to feed through power. The chamber must be opened to ensure correct mating to the access ports and validation of the connections. Once these are in place, the sub-stage can be modified and iterated on without the need for chamber venting. This system as well as subsequent microthermocouple testing will provide a test bed for development of a commercially viable solution for other SEM tools.

\section{ACKNOWLEDGMENTS}

Acknowledgements. We are grateful for partial support via Discovery grants DP120102980 and DP130101205 from the Australian Research Council (ARC). We acknowledge discussions on ion-induced surface charging with Dr. Paul Spizzirri.

\section{REFERENCES}

[1] Postek, M. T. and Vladár, A. E., "Nanomanufacturing concerns about measurements made in the SEM part IV: charging and its mitigation," in [Nanoeng. Fabr. Prop. Opt. Devices XII], Campo, E. M., Dobisz, E. A., and Eldada, L. A., eds., 9556, 95560Q (aug 2015).

[2] Gervinskas, G., Seniutinas, G., and Juodkazis, S., "Control of surface charge for high-fidelity nanostructuring of materials," Laser Photon. Rev. 7, 1049-1053 (nov 2013).

[3] Seniutinas, G., Balčytis, A., and Juodkazis, S., "Ultraviolet-photoelectric effect for augmented contrast and resolution in electron microscopy," APL Photonics 1(2), 021301 (2016).

[4] Juodkazis, S. and Rosa, L., "Surface defect mediated electron hopping between nanoparticles separated by a nano-gap," Phys. Status Solidi - Rapid Res. Lett. 4(10), 244-246 (2010). 
[5] Efimov, O., Juodkazis, S., and Misawa, H., "Intrinsic single- and multiple-pulse laser-induced damage in silicate glasses in the femtosecond-to-nanosecond region," Phys. Rev. A - At. Mol. Opt. Phys. 69(4), 1-7 (2004).

[6] Saeta, P. N. and Greene, B. I., "Primary relaxation processes at the band edge of SiO2," Phys. Rev. Lett. 70(23), 3588-3591 (1993).

[7] Pollack, S. E., Turner, M. D., Schlamminger, S., Hagedorn, C. A., and Gundlach, J. H., "Charge management for gravitational-wave observatories using UV LEDs," Phys. Rev. D 81, 021101 (jan 2010).

[8] Balčytis, A., Ryu, M., Juodkazis, S., and Morikawa, J., "Micro-thermocouple on nano-membrane: thermometer for nanoscale measurements," Sci. Rep. 8, 6324 (dec 2018). 